\title{
ADAPTIVE SELECTION OF PRIMAL CONSTRAINTS FOR ISOGEOMETRIC BDDC DELUXE PRECONDITIONERS*
}

\author{
L. BEIRÃO DA VEIGA ${ }^{\dagger}$, L. F. PAVARINO ${ }^{\ddagger}$, S. SCACCHI $^{\ddagger}$, O. B. WIDLUND SAND $^{\S}$ AND
}

\author{
S. ZAMPINI
}

\begin{abstract}
Isogeometric analysis has been introduced as an alternative to finite element methods in order to simplify the integration of computer-aided design (CAD) software and the discretization of variational problems of continuum mechanics. In contrast with the finite element case, the basis functions of isogeometric analysis are often not nodal. As a consequence, there are fat interfaces which can easily lead to an increase in the number of interface variables after a decomposition of the parameter space into subdomains. Building on earlier work on the deluxe version of the BDDC (balancing domain decomposition by constraints) family of domain decomposition algorithms, several adaptive algorithms are developed in this paper for scalar elliptic problems in an effort to decrease the dimension of the global, coarse component of these preconditioners. Numerical experiments provide evidence that this work can be successful, yielding scalable and quasi-optimal adaptive $\mathrm{BDDC}$ algorithms for isogeometric discretizations.
\end{abstract}

Key words. domain decomposition, BDDC deluxe preconditioners, isogeometric analysis, adaptive primal constraints, elliptic problems

AMS subject classifications. 65F08, 65N30, 65N35, 65N55

DOI. $10.1137 / 15 \mathrm{M} 1054675$

1. Introduction. There has recently been considerable effort toward developing adaptive methods for the selection of primal constraints for BDDC (balancing domain decomposition by constraints) algorithms, including its deluxe variant. The primal constraints of a BDDC or FETI-DP (dual-primal finite element tearing and interconnect) algorithm provide the global, coarse part of such a preconditioner and are of crucial importance for obtaining rapid convergence of these preconditioned conjugate gradient methods for the case of many subdomains. When the primal constraints are chosen adaptively, we aim at selecting a primal space, which for a certain dimension of the coarse space provides the fastest rate of convergence for the iterative method. In the alternative, we can try to develop criteria which will guarantee that the condition number of the iteration stays below a given tolerance.

In this paper, we will consider the use of adaptive algorithms to select the primal constraints and the associated BDDC change of basis for elliptic problems and isogeometric analysis. While for lower order finite element approximations one typically starts out with a small primal space, associated with all the subdomain vertex vari-

* Submitted to the journal's Methods and Algorithms for Scientific Computing section December 28, 2015; accepted for publication (in revised form) October 26, 2016; published electronically February 23, 2017.

http://www.siam.org/journals/sisc/39-1/M105467.html

Funding: The work of the first three authors was supported by grants from M.I.U.R. (PRIN 201289A4LX_002) and from the Istituto Nazionale di Alta Matematica (INDAM-GNCS). The work of the fourth author was partially supported by National Science Foundation grant DMS-1522736.

${ }^{\dagger}$ Dipartimento di Matematica e Applicazioni, Università degli Studi di Milano-Bicocca, Via Cozzi, 53, 20125 Milano, Italy (lourenco.beirao@unimib.it).

‡Dipartimento di Matematica, Università degli Studi di Milano, Via Saldini 50, 20133 Milano, Italy (luca.pavarino@unimi.it, simone.scacchi@unimi.it).

$\S$ Courant Institute of Mathematical Sciences, 251 Mercer Street, New York, NY 10012 (widlund@ cims.nyu.edu, http://cs.nyu.edu/cs/faculty/widlund/index.html).

ฯExtreme Computing Research Center, King Abdullah University of Science and Technology, Thuwal 23955, Saudi Arabia (stefano.zampini@kaust.edu.sa).

A281 
ables, and then adds primal constraints in order to obtain improved iteration counts, a similar strategy for an isogeometric problem will introduce a primal space that can be quite large, especially if we have a high polynomial degree and high regularity inside the patches. This depends on the fact that we have fat vertices, potentially with many degrees of freedom, as well as fat edges and faces, resulting from the fact that the basis functions are not nodal and can have large supports; see section 3 for a definition of these fat interface classes. Therefore, in this study, we will attempt to reduce this original primal space by developing adaptive algorithms. We will also consider subspaces of primal variables associated with the subdomain edges and, in three dimensions, subdomain faces. We note that we made an attempt to shrink the primal spaces in a previous study [7], but that our results then deteriorated when the degree of the basis functions grew. At that time, we did not have tools for the automatic selection of our primal space. We also note that the linear systems resulting from discretizing the elliptic problems using isogeometric analysis become extremely ill-conditioned with increasing polynomial degree and high regularity (see [20]), and therefore the development of preconditioners is quite challenging. For an introduction to isogeometric analysis, see [12], and for a recent survey of the state of the theory, see [3]. For earlier work on preconditioned iterative solvers for isogeometric approximations, see [4, 6, 8, 11, 19,30]; see also [36] for a recent extension of dual-primal preconditioners to isogeometric discretizations of almost incompressible elasticity and Stokes problems, partly based on work by Tu and Li [41].

The work on adaptive selection of primal constraints over the last few years has focused on lower order finite elements, and several of these methods are now fully justified theoretically and also perform very satisfactorily. Until quite recently, the development of the theory has been focused on primal constraints for equivalence classes with two elements, such as those of subdomain edges for problems defined on domains in the plane; see, e.g., a recent survey paper by Klawonn, Radtke, and Rheinbach [28]. For other papers on this case, see [15, 22, 25, 26, 27, 34, 37, 39]. Most of these papers focus on the adaptive selection of two-dimensional (2D) or three-dimensional (3D) face constraints, i.e., constraints associated with the interface between pairs of subdomains, by solving certain generalized eigenproblems. While it is important to further study the best way of handling all cases, the basic issues appear to be well settled when the equivalence classes have no more than two elements. We note that, in our context, an equivalence class is a set of spline knots, associated with basis functions the supports of which intersect the same set of subdomains into which the parameter space has been subdivided; see sections 2 and 3.

For problems in three dimensions, there is, except for quite special subdomain configurations, a need to develop algorithms and results for equivalence classes with three or more elements. There is work by Mandel, Sousedík, and Šístek, who developed condition number indicators in [34]. Talks by Dohrmann and by Klawonn at the Twenty-third International Conference on Domain Decomposition Methods (DD23, July, 2015) reported on considerable progress in giving similar algorithms a firm theoretical basis and have since resulted in [38] and [24]. A talk by Kim at the same conference, on joint work with Chung and Wang [23], also reported considerable progress. Their main algorithm for problems in three dimensions is similar to but not the same as that of [10]. The main results of the latter paper, which were developed independently, were also reported at the same DD23 minisymposium by the second author of [10]. We note that the paper by Pechstein and Dohrmann [38] presents a unified approach to all of these methods. As we have already noted, for isogeometric problems, there is a need to consider these issues even for the fat subdomain vertices 
which are associated with more than two subdomains, even for 2D problems.

We note that in the design of a BDDC algorithm we have to choose a primal space and also an average operator associated with the interface between the subdomains into which the given domain of the elliptic problem has been subdivided. The choice of primal constraints is the main issue of this paper, and we will explore a number of different choices. As for the averaging, based on our experience in a previous study [7], we will exclusively use the deluxe variant of BDDC; for older, less competitive algorithms, see [5].

The rest of this paper is organized as follows: A brief overview of isogeometric analysis is given in section 2. This is followed by a short introduction to BDDC, including its deluxe variant, in section 3. The subsequent section introduces several generalized eigenvalue problems with which we can make adaptive choices of primal constraints for our BDDC algorithms. In section 5, numerical results are provided for several different adaptive algorithms, which with quite small primal constraint spaces result in a small number of iterations even for problems based on splines of high order and high regularity.

2. Isogeometric discretization of scalar elliptic problems. Given a bounded and connected domain $\Omega \subset \mathbb{R}^{d}, d=2,3$, typically generated by a CAD (computeraided design) software system, we consider the model elliptic problem

$$
-\nabla \cdot(\rho \nabla u)=f \quad \text { in } \Omega, \quad u=0 \quad \text { on } \partial \Omega,
$$

with a scalar coefficient $\rho$ satisfying $0<\rho_{\min } \leqslant \rho(x) \leqslant \rho_{\max } \forall x \in \Omega$. For simplicity, we describe our problem and preconditioner mostly for the $2 \mathrm{D}$ single-patch case. Comments on extensions to three dimensions and multipatches can be found in [7].

We discretize (2.1) with isogeometric analysis (IGA) techniques based on B-splines and nonuniform rational basis splines (NURBS) basis functions; see, e.g., [21] for a general introduction to IGA. The bivariate B-spline discrete space is defined by

$$
\widehat{\mathcal{S}}_{h}:=\operatorname{span}\left\{B_{i, j}^{p, q}(\xi, \eta), i=1, \ldots, n, j=1, \ldots, m\right\},
$$

where the bivariate B-spline basis functions $B_{i, j}^{p, q}(\xi, \eta):=N_{i}^{p}(\xi) M_{j}^{q}(\eta)$ are defined by tensor products of $1 \mathrm{D}$ B-spline functions $N_{i}^{p}(\xi)$ and $M_{j}^{q}(\eta)$ of degree $p$ and $q$, respectively; in our numerical experiments, we will consider only the case of $p=q$. An additional important parameter is $k \leqslant p-1$, the number of continuous derivatives of the basis functions. In the parameter space, there is a tensor product mesh with rectangular elements associated with the B-spline knots; we will also define our subdomains in terms of rectangles formed by unions of such elements.

Analogously, the NURBS space is the span of NURBS basis functions defined in one dimension by

$$
R_{i}^{p}(\xi):=\frac{N_{i}^{p}(\xi) \omega_{i}}{\sum_{k=1}^{n} N_{k}^{p}(\xi) \omega_{k}}=\frac{N_{i}^{p}(\xi) \omega_{i}}{w(\xi)},
$$

with a positive weight function $w(\xi):=\sum_{k=1}^{n} N_{k}^{p}(\xi) \omega_{k} \in \widehat{\mathcal{S}}_{h}$, and in two dimensions by

$$
R_{i, j}^{p, q}(\xi, \eta):=\frac{B_{i, j}^{p, q}(\xi, \eta) \omega_{i, j}}{\sum_{k=1}^{n} \sum_{\ell=1}^{m} B_{k, \ell}^{p, q}(\xi, \eta) \omega_{k, \ell}}=\frac{B_{i, j}^{p, q}(\xi, \eta) \omega_{i, j}}{w(\xi, \eta)},
$$

where $w(\xi, \eta)$ is the weight function and $\omega_{i, j}$ the positive weights associated with a $n \times m$ net of control points given by $\mathbf{C}_{i, j}$. The discrete space of NURBS functions on 
the domain $\Omega$ is defined as the span of the push-forward of the NURBS basis functions (2.4) (see, e.g., [21]),

$$
\mathcal{N}_{h}:=\operatorname{span}\left\{R_{i, j}^{p, q} \circ \mathbf{F}^{-1} \text {, with } i=1, \ldots, n, j=1, \ldots, m\right\},
$$

with $\mathbf{F}: \widehat{\Omega} \rightarrow \Omega$, the geometrical map between parameter and physical spaces, defined by $\mathbf{F}(\xi, \eta):=\sum_{i=1}^{n} \sum_{j=1}^{m} R_{i, j}^{p, q}(\xi, \eta) \mathbf{C}_{i, j}$.

For simplicity, we will consider only the case with a Dirichlet boundary condition imposed on all of $\partial \Omega$, and we can then define the spline space in the parameter space and the NURBS space in physical space, respectively, as

$$
\begin{gathered}
\widehat{V}_{h}:=\widehat{\mathcal{S}}_{h} \cap H_{0}^{1}(\widehat{\Omega})=\operatorname{span}\left\{B_{i, j}^{p, q}(\xi, \eta), i=2, \ldots, n-1, j=2, \ldots, m-1\right\}, \\
V_{h}:=\mathcal{N}_{h} \cap H_{0}^{1}(\Omega)=\operatorname{span}\left\{R_{i, j}^{p, q} \circ \mathbf{F}^{-1}, i=2, \ldots, n-1, j=2, \ldots, m-1\right\} .
\end{gathered}
$$

The IGA formulation of problem (2.1) then reads

$$
\left\{\begin{array}{l}
\text { Find } u_{h} \in V_{h} \text { such that } \\
a\left(u_{h}, v_{h}\right)=\left\langle f, v_{h}\right\rangle \quad \forall v_{h} \in V_{h},
\end{array}\right.
$$

with the bilinear form $a\left(u_{h}, v_{h}\right):=\int_{\Omega} \rho \nabla u_{h} \cdot \nabla v_{h} d x$ and right-hand side $\left\langle f, v_{h}\right\rangle:=$ $\int_{\Omega} f v_{h} d x$.

3. Equivalence classes, Schur complements, and BDDC preconditioners. After introducing the equivalence classes relevant for isogeometric approximations (see also [7]), we will give a short introduction to BDDC algorithms; for more details, see, e.g., [31]. For an introduction to its deluxe variant, see, e.g., [42].

BDDC algorithms are domain decomposition algorithms based on the decomposition of the domain $\Omega$ of an elliptic operator into nonoverlapping subdomains $\Omega_{i}$. Such algorithms were introduced by Dohrmann in 2003 [14], a few years after the introduction of the FETI-DP algorithms; see [18]. Important theoretical findings are given in $[32,33]$. In the case of lower order finite elements, each of the subdomains is often associated with tens of thousands of degrees of freedom. The subdomain interface $\Gamma_{i}$ of $\Omega_{i}$ does not cut through any elements and is defined by $\Gamma_{i}:=\partial \Omega_{i} \backslash \partial \Omega$. In the isogeometric $2 \mathrm{D}$ context, the subdomain $\Omega_{i}$ are images of rectangles $\widehat{\Omega}_{i}$ in the parameter space, each a union of rectangular elements defined by four knots, which form its vertices.

The equivalence classes associated with the subdomains are defined as follows for 3D problems: We first separate the knots of the interior of these subdomains and those associated with the interface $\Gamma:=\bigcup_{i} \Gamma_{i}$; those in the interior are the knots with B-spline basis functions supported in individual subdomains. The set of the remaining interface knots does not form just a skeleton as for low order finite elements, but forms a "fat" interface due to the large support of the spline basis functions (see, e.g., [7, Figure 1]). We partition this fat interface into equivalence classes associated with subdomain vertices, edges, and faces. We first separate off the fat vertex equivalence sets, which are given by the knots with B-spline basis functions with a subdomain vertex inside their support. We next identify the fat edge equivalence classes among the remaining interface knots with B-spline basis functions with supports that intersect a subdomain edge. Finally, the remaining fat interface knots, which have basis functions with supports intersecting a subdomain face, are separated into subsets associated with the individual subdomain faces. Once 
these equivalence classes have been identified, we will find many similarities with the development of BDDC algorithms for finite element problems.

Given the stiffness matrix $A^{(i)}$ of the subdomain $\Omega_{i}$ and its part of the fat interface, we obtain a subdomain Schur complement $S^{(i)}$ by eliminating the interior variables, i.e., all those associated with basis functions with supports confined to $\Omega_{i}$. We will also work with principal minors of these Schur complements associated with a subdomain vertex, subdomain edge, and subdomain face, denoting them by $S_{V V}^{(i)}, S_{E E}^{(i)}$, and $S_{F F}^{(i)}$, respectively.

The interface space is then divided into a primal subspace of functions which are continuous and a complementary, dual, subspace for which we will allow multiple values across the interface during part of each iteration step. The BDDC and FETI-DP algorithms can be described in terms of three product spaces of functions associated with sets of interface knots:

$$
\widehat{W}_{\Gamma} \subset \widetilde{W}_{\Gamma} \subset W_{\Gamma} .
$$

$W_{\Gamma}$ is built as a product space of the spaces associated with the $\Gamma_{i}$, without any continuity constraints across the interface. Elements of $\widetilde{W}_{\Gamma}$ have common values of the primal variables but allow multiple values of the dual variables, while the elements of $\widehat{W}_{\Gamma}$ are continuous at all knots of $\Gamma$. We can change variables, explicitly introducing the primal variables and complementary sets of dual variables. This simplifies the presentation and also appears to make the methods more robust; alternative ways of implementing the algorithms are also possible (see, e.g., [14]). After eliminating the interior variables, we can then write the subdomain Schur complements as

$$
S^{(i)}=\left(\begin{array}{cc}
S_{\Delta \Delta}^{(i)} & S_{\Delta \Pi}^{(i)} \\
S_{\Pi \Delta}^{(i)} & S_{\Pi \Pi}^{(i)}
\end{array}\right) .
$$

We will partially subassemble the $S^{(i)}$, obtaining $\breve{S}$, enforcing the continuity of the primal variables only. Thus, we then work in $\widetilde{W}_{\Gamma_{\dot{S}}}$ In each step of the iteration, we solve a linear system with the coefficient matrix $\breve{S}$. In the alternative, we could also work with a linear system with a matrix obtained by partially subassembling the subdomain stiffness matrices $A^{(i)}$. We note that solving these linear systems will be much faster than if we work with the fully assembled system if the dimension of the primal space is modest. At the end of each iteration, the approximate solution is made continuous at all knots of the interface; continuity is restored by applying a weighted averaging operator $E_{D}$, which maps $\bar{W}_{\Gamma}$ into $\widehat{W}_{\Gamma}$.

In each iteration, we first compute the residual of the fully assembled Schur complement. We then apply $E_{D}^{T}$ to obtain a right-hand side of the partially subassembled linear system, solve this system, and then apply $E_{D}$. This last step changes the values on $\Gamma$, unless the iteration has converged, and can result in nonzero residuals at interior knots next to $\Gamma$. In a final step of each iteration step, we eliminate these residuals by solving a Dirichlet problem on each of the subdomains. We always accelerate the iteration with the preconditioned conjugate gradient algorithm.

3.1. BDDC deluxe. When designing a BDDC algorithm, we have to choose an effective set of primal constraints and also a good recipe for the averaging across the interface. This paper concerns the choice of the primal constraints, while we will always use the deluxe recipe in the construction of the averaging operator $E_{D}$.

In work on $3 \mathrm{D}$ problems formulated in $H$ (curl), it was found that traditional averaging recipes did not work uniformly well; cf. $[16,17]$. The same is true for 
problems in $H$ (div); see [35]. This occasional failure has its roots in the fact that there are two sets of material parameters in these applications. The deluxe variant that was then introduced has proven quite successful in a variety of applications; see, e.g., [42] and, in particular, [7].

A face component of the average operator $E_{D}$, for a problem in three dimensions, across a subdomain face $F \subset \Gamma$, common to two subdomains $\Omega_{i}$ and $\Omega_{j}$, is defined in terms of that face equivalence set of variables and principal minors $S_{F F}^{(k)}$ of the $S^{(k)}$, $k=i, j$. The deluxe averaging operator, for $F$, is then defined by

$$
\bar{w}_{F}:=\left(E_{D} w\right)_{F}:=\left(S_{F F}^{(i)}+S_{F F}^{(j)}\right)^{-1}\left(S_{F F}^{(i)} w_{F}^{(i)}+S_{F F}^{(j)} w_{F}^{(j)}\right) .
$$

Here $w_{F}^{(i)}$ is the restriction of $w^{(i)}$ to the face set, $F$ (and analogously for $w_{F}^{(j)}$ ). By replacing $F$ by $E$, we obtain the formula for an edge for a 2D problem.

The action of $\left(S_{F F}^{(i)}+S_{F F}^{(j)}\right)^{-1}$ can be implemented by solving a Dirichlet problem, with zero boundary values, on $\Omega_{i} \cup F \cup \Omega_{j}$, where $F$ is the face between the two subdomains and with a right-hand side which vanishes in the interior of the two subdomains. This can add significantly to the cost. In the economic version $(e-$ version), we replace this large domain by a thin domain built from one or a few layers of elements next to the face, and this often results in very similar performance; see, e.g., $[17]$.

Deluxe averaging operators are also developed for subdomain edges and subdomain vertices for problems in three dimensions and for subdomain vertices alone for problems in two dimensions. Given the simple geometry of the parameter space that we are considering, we find that in all these cases the equivalence classes will have four or eight elements for any subdomain vertex or edge in the interior of $\Omega$. Thus, for an interior subdomain vertex $V$ in two dimensions, shared by subdomains $\Omega_{i}, \Omega_{j}, \Omega_{k}, \Omega_{l}$, we will use the formula

$$
\bar{w}_{V}:=\left(S_{V V}^{(i)}+S_{V V}^{(j)}+S_{V V}^{(k)}+S_{V V}^{(\ell)}\right)^{-1}\left(S_{V V}^{(i)} w_{V}^{(i)}+S_{V V}^{(j)} w_{V}^{(j)}+S_{V V}^{(k)} w_{V}^{(k)}+S_{V V}^{(\ell)} w_{V}^{(\ell)}\right) .
$$

The core of any estimate for a BDDC algorithm involves the norm of the average operator $E_{D}$. By an algebraic argument known, for FETI-DP, since 2002 (cf. [29]), we know that

$$
\operatorname{cond}\left(M_{B D D C}^{-1} \widehat{S}\right) \leqslant\left\|E_{D}\right\|_{\breve{S}},
$$

where $M_{B D D C}^{-1}$ is the BDDC preconditioner and $\widehat{S}$ is the Schur complement of the stiffness matrix $A$ of the discrete problem (2.6). We recall that FETI-DP and BDDC methods with the same set of primal constraints have essentially the same spectrum, except for possible 0 and 1 eigenvalues; see [33, 31, 9].

4. Condition number bounds and generalized eigenvalue problems. We will base our discussion, in part, on results recently developed in [10]. But we will first follow Dohrmann and Pechstein closely; cf. [15, 37]. They managed to simplify a relevant expression for the case of equivalence classes with two elements and also found an interesting old reference [1] in which parallel sums were introduced; these ideas have also been explored by Klawonn et al. [25] and by Kim, Chung, and Wang [23] .

For any two symmetric positive definite matrices $A$ and $B$, we define their parallel sum as

$$
A: B:=\left(A^{-1}+B^{-1}\right)^{-1} \text {. }
$$


This is relevant for a face in three dimensions (or an edge in two dimensions) since, for equivalence classes with two elements, the relevant generalized eigenvalue problem will, as is convincingly argued in [38, subsection 5.2], be

$$
\widetilde{S}_{F F}^{(i)}: \widetilde{S}_{F F}^{(j)} \phi=\lambda S_{F F}^{(i)}: S_{F F}^{(j)} \phi .
$$

Here the matrix on the right, built from principal minors of $S^{(i)}$ and $S^{(j)}$, respectively, will be strictly positive definite. The matrices on the left of (4.2) represent the minimal energy extension of the values on the face $F$ onto the rest of $\Gamma_{i}$. Writing a subdomain Schur complement as

$$
S^{(i)}=\left(\begin{array}{ll}
S_{F^{\prime} F^{\prime}}^{(i)} & S_{F^{\prime} F}^{(i)} \\
S_{F^{\prime} F}^{(i) T} & S_{F F}^{(i)}
\end{array}\right),
$$

where $F^{\prime}:=\Gamma_{i} \backslash F$, we find that

$$
\widetilde{S}_{F F}^{(i)}:=S_{F F}^{(i)}-S_{F^{\prime} F}^{(i) T} S_{F^{\prime} F^{\prime}}^{(i)-1} S_{F^{\prime} F}^{(i)} .
$$

These matrices are only positive semidefinite for subdomains in the interior of the domain $\Omega$ if there are no existing constraints. We can handle any such singular matrices by modifying the definition of the parallel sum using generalized inverses or by adding to the Schur complements $S^{(i)}$ and $S^{(j)}$ the term $\epsilon I$, with $\epsilon>0$ small compared with the eigenvalues of $S^{(i)}$ and $S^{(j)}$. Any existing constraint, e.g., a nonet-flux condition for the Darcy flow or for almost incompressible elasticity, can also be incorporated, and additional constraints can be obtained by projecting (4.2) onto the space orthogonal to the existing constraints [46]. For additional insight, see [38].

Instead of developing an estimate for $E_{D}$, we will work with $P_{D}:=I-E_{D}$. Thus, we estimate the $S^{(i)}$-norm of $R_{F}^{T}\left(w_{F}^{(i)}-\bar{w}_{F}\right)$, instead of $\left(R_{F}^{T} \bar{w}_{F}\right)^{T} S^{(i)} R_{F}^{T} \bar{w}_{F}$. Here $R_{F}$ denotes the restriction to the face $F$. By simple algebra, we find that

$$
w_{F}^{(i)}-\bar{w}_{F}=\left(S_{F F}^{(i)}+S_{F F}^{(j)}\right)^{-1} S_{F F}^{(j)}\left(w_{F}^{(i)}-w_{F}^{(j)}\right) .
$$

More algebra gives, by using that $S_{F F}^{(i)}:=R_{F} S^{(i)} R_{F}^{T}$,

$$
\begin{aligned}
& \left(R_{F}^{T}\left(w_{F}^{(i)}-\bar{w}_{F}\right)\right)^{T} S^{(i)}\left(R_{F}^{T}\left(w_{F}^{(i)}-\bar{w}_{F}\right)\right) \\
& \quad=\left(w_{F}^{(i)}-w_{F}^{(j)}\right)^{T} S_{F F}^{(j)}\left(S_{F F}^{(i)}+S_{F F}^{(j)}\right)^{-1} S_{F F}^{(i)}\left(S_{F F}^{(i)}+S_{F F}^{(j)}\right)^{-1} S_{F F}^{(j)}\left(w_{F}^{(i)}-w_{F}^{(j)}\right) .
\end{aligned}
$$

Adding a similar contribution from $\Omega_{j}$, we obtain, following Pechstein and Dohrmann [37], that the relevant expression of the energy is

$\left(w_{F}^{(i)}-w_{F}^{(j)}\right)^{T}\left(S_{F F}^{(i)^{-1}}+S_{F F}^{(j)^{-1}}\right)^{-1}\left(w_{F}^{(i)}-w_{F}^{(j)}\right)=\left(w_{F}^{(i)}-w_{F}^{(j)}\right)^{T}\left(S_{F F}^{(i)}: S_{F F}^{(j)}\right)\left(w_{F}^{(i)}-w_{F}^{(j)}\right)$,

which can easily be bounded by

$$
2 w_{F \Delta}^{(i) T}\left(S_{F F}^{(i)}: S_{F F}^{(j)}\right) w_{F \Delta}^{(i)}+2 w_{F \Delta}^{(j) T}\left(S_{F F}^{(i)}: S_{F F}^{(j)}\right) w_{F \Delta}^{(j)} .
$$

Here $w_{F \Delta}^{(i)}:=w_{F}^{(i)}-w_{\Pi}$, etc., and $w_{\Pi}$ is an arbitrary element of the primal space.

There now remains to estimate $w_{F \Delta}^{(i) T}\left(S_{F F}^{(i)}: S_{F F}^{(j)}\right) w_{F \Delta}^{(i)}$ by the energy of $w^{(i)}$. We do so by establishing a bound for

$$
w_{F \Delta}^{(i) T}\left(S_{F F}^{(i)}: S_{F F}^{(j)}\right) w_{F \Delta}^{(i)} \quad \text { by } \quad w_{F \Delta}^{(i) T}\left(\widetilde{S}_{F F}^{(i)}: \widetilde{S}_{F F}^{(j)}\right) w_{F \Delta}^{(i)} .
$$

Copyright $@$ by SIAM. Unauthorized reproduction of this article is prohibited. 
This will be accomplished by considering the related Rayleigh quotient restricted to a subspace given by a span of eigenvectors of the generalized eigenvalue problem (4.2). We will finally show that

$$
w_{F \Delta}^{(i) T}\left(\widetilde{S}_{F F}^{(i)}: \widetilde{S}_{F F}^{(j)}\right) w_{F \Delta}^{(i)} \leqslant w^{(i) T} S^{(i)} w^{(i)},
$$

where $w^{(i)}$ is an arbitrary extension of the values of $w_{F}^{(i)}$ on the face $F$ to the rest of $\Gamma_{i}$.

In standard BDDC theory, the estimate related to (4.5) can be obtained by using a face lemma; cf. [40, subsection 4.6.3], where such a result is established for low order finite elements, constant coefficients in each subdomain, and polyhedral subdomains. For an adaptive algorithm, such a result is replaced by using the generalized eigenvalue problem (4.2). Thus, we generate elements of the primal and dual spaces for $F$ by solving the generalized eigenvalue problem (4.2). Primal constraints are then generated by making the eigenvectors of a few of the smallest eigenvalues of (4.2) orthogonal to $\left(\widetilde{S}_{F F}^{(i)}: \widetilde{S}_{F F}^{(j)}\right)\left(w_{F}^{(i)}-w_{F}^{(j)}\right)$; see section 4.2 below for more details and two possible strategies. The dual space can be defined by the span of the remaining eigenvectors making the primal and dual spaces orthogonal in the inner product defined by $\widetilde{S}_{F F}^{(i)}: \widetilde{S}_{F F}^{(j)}$. This orthogonality condition allows us to conclude that

$$
w_{F \Delta}^{(i) T}\left(\widetilde{S}_{F F}^{(i)}: \widetilde{S}_{F F}^{(j)}\right) w_{F \Delta}^{(i)} \leqslant w_{F}^{(i) T}\left(\widetilde{S}_{F F}^{(i)}: \widetilde{S}_{F F}^{(j)}\right) w_{F}^{(i)} .
$$

We now use that, trivially, $\widetilde{S}_{F F}^{(i)}: \widetilde{S}_{F F}^{(j)} \leqslant \widetilde{S}_{F F}^{(i)}$ and find that

$$
w_{F}^{(i) T}\left(\widetilde{S}_{F F}^{(i)}: \widetilde{S}_{F F}^{(j)}\right) w_{F}^{(i)} \leqslant w_{F}^{(i) T} \widetilde{S}_{F F}^{(i)} w_{F}^{(i)} \leqslant w^{(i) T} S^{(i)} w^{(i)}
$$

for any $w^{(i)}$ which coincides with $w_{F}^{(i)}$ on $F$. Here we use the fact that $\widetilde{S}_{F F}^{(i)}$ provides the minimum energy extension of the values of $w_{F}^{(i)}$.

A bound can now be obtained in terms of the smallest eigenvalue with an eigenvector not used in deriving the primal constraints. Thus, if $\lambda_{t o l}$ is the smallest eigenvalue of (4.2) ignored when we select the primal variables, we obtain the bound

$$
\left\|\left(P_{D} w\right)_{\mid F}\right\|_{\breve{S}}^{2} \leqslant \frac{2}{\lambda_{t o l}}\left(w^{(i) T} S^{(i)} w^{(i)}+w^{(j) T} S^{(j)} w^{(j)}\right) .
$$

We note that the factor 2 originates from (4.4), and the other factor from the bound related to (4.5).

4.1. Generalized eigenvalue problems for equivalence classes with more than two elements. We will now attempt to derive primal constraints by using generalized eigenvalue problems for equivalence classes with four elements; this is relevant for our special application since a subdomain vertex is common to four subdomains in two dimensions, as is a subdomain edge in three dimensions. We note that in three dimensions an interior subdomain vertex is common to eight subdomains. We also note that the case of equivalence classes with three elements is discussed in detail in [10], and that there are no essential differences in the development of theory and practice for equivalence classes with three or more elements.

Let us focus on the case of a subdomain vertex $V$ in two dimensions. We will consider a number of algorithms built from $S_{V V}^{(i)}$ and $\widetilde{S}_{V V}^{(i)}$, where $i$ will take on the values of the indices of the four subdomains, which have $V$ in common. We will first 
derive an algorithm that can be fully justified and which works well for 3D lower order finite element problems; see [10].

An expression for the norm of $R_{V}^{T}\left(w_{V}^{(i)}-\bar{w}_{V}\right)$ can be borrowed from [10]. We find that the sum of the squares of the $S^{(i)}$-norms of $R_{V}^{T} P_{D} w$, for the four relevant indices, can be estimated from above by the sum of four terms, the first two of which are

and

$$
4 w_{V}^{(i) T} S_{V V}^{(i)}:\left(S_{V V}^{(j)}+S_{V V}^{(k)}+S_{V V}^{(\ell)}\right) w_{V}^{(i)}
$$

$$
4 w_{V}^{(j) T} S_{V V}^{(j)}:\left(S_{V V}^{(i)}+S_{V V}^{(k)}+S_{V V}^{(\ell)}\right) w_{V}^{(j)} .
$$

Thus, with four subdomains in the equivalence class, there are four operators $T_{V}^{(i)}:=S_{V V}^{(i)}:\left(S_{V V}^{(j)}+S_{V V}^{(k)}+S_{V V}^{(\ell)}\right), T_{V}^{(j)}:=S_{V V}^{(j)}:\left(S_{V V}^{(i)}+S_{V V}^{(k)}+S_{V V}^{(\ell)}\right)$ (and analogously for $\left.T_{V}^{(k)}, T_{V}^{(l)}\right)$. These operators are symmetric positive definite, and they appear directly in our estimate of the energy of $\left(P_{D} w\right)_{\mid V}$. We can now use the trivial inequality

$$
w_{V}^{(i) T} T_{V}^{(i)} w_{V}^{(i)} \leqslant w_{V}^{(i) T}\left(T_{V}^{(i)}+T_{V}^{(j)}+T_{V}^{(k)}+T_{V}^{(\ell)}\right) w_{V}^{(i)}
$$

and very similar bounds for the other terms and arrive at the generalized eigenvalue problem

$$
\left(\widetilde{S}_{V V}^{(i)}: \widetilde{S}_{V V}^{(j)}: \widetilde{S}_{V V}^{(k)}: \widetilde{S}_{V V}^{(\ell)}\right) \phi=\lambda\left(T_{V}^{(i)}+T_{V}^{(j)}+T_{V}^{(k)}+T_{V}^{(\ell)}\right) \phi
$$

Suitable primal constraints can now be obtained from the eigenvectors associated with the smallest eigenvalues of (4.7) in the same way as in the previous subsection. The resulting primal space will be denoted by $V_{T}$ below (see (4.10)) and in the numerical tests. A justification for this algorithm can then be obtained by developing a bound of the same nature as (4.6) and combining these two sets of bounds. The result will involve integer factors depending on the square of the maximal number of vertices and edges of the subdomains. We note that in many cases the condition numbers actually observed in numerical experiments are much smaller than what can now be established theoretically.

4.2. Alternative generalized eigenvalue problems. In addition to the algorithm derived in the previous subsection, we have also experimented with several alternative generalized eigenvalue problems. They are all defined in terms of the Schur complements $S^{(i)}$ and $\widetilde{S}^{(i)}$ introduced early on in section 4 , and we will build the matrices of the generalized eigenvalue problems from these matrices, their sums, and their parallel sums. These alternative algorithms cannot, so far, be justified to the same extent as the algorithm of subsection 4.1.

In order to simplify the description of our strategy, we will consider the case of an equivalence class related to four subdomains, such as for a fat vertex in two dimensions or a fat edge in 3D structured hexahedral subdomain meshes.

Generalized eigenproblem $V_{1}$ :

$$
\widetilde{S}_{V V}^{(k)} \phi=\lambda S_{V V}^{(k)} \phi,
$$

where $k$ is any of the indices of the subdomains sharing the edge. This is a minimal choice involving only a pair of associated Schur complements instead of several pairs as in the choices below.

Generalized eigenproblem $V_{\text {par }}$ :

$$
\left(\widetilde{S}_{V V}^{(i)}: \widetilde{S}_{V V}^{(j)}: \widetilde{S}_{V V}^{(k)}: \widetilde{S}_{V V}^{(\ell)}\right) \phi=\lambda\left(S_{V V}^{(i)}: S_{V V}^{(j)}: S_{V V}^{(k)}: S_{V V}^{(\ell)}\right) \phi .
$$

Copyright $@$ by SIAM. Unauthorized reproduction of this article is prohibited. 
This choice is the natural extension to four subdomains of the parallel sum eigenproblem (4.2) defined in the case of two subdomains; this recipe has been shown to produce robust primal spaces for heterogeneous $H(\mathbf{c u r l})$ problems in three dimensions with tetrahedral meshes [44, 45].

Generalized eigenproblem $V_{T}$ :

$$
\left(\widetilde{S}_{V V}^{(i)}: \widetilde{S}_{V V}^{(j)}: \widetilde{S}_{V V}^{(k)}: \widetilde{S}_{V V}^{(\ell)}\right) \phi=\lambda\left(T_{V}^{(i)}+T_{V}^{(j)}+T_{V}^{(k)}+T_{V}^{(\ell)}\right) \phi
$$

where $T_{V}^{(i)}=S_{V V}^{(i)}:\left(S_{V V}^{(j)}+S_{V V}^{(k)}+S_{V V}^{(\ell)}\right), \ldots, T_{V}^{(\ell)}=S_{V V}^{(\ell)}:\left(S_{V V}^{(i)}+S_{V V}^{(j)}+S_{V V}^{(k)}\right)$. This is the same as (4.7) in the previous section, and it is motivated by the theoretical analysis presented in section 4.1, which we were able to carry out only for this specific choice of $T_{V}^{(i)}$ operators.

Generalized eigenproblem $V_{m i x}$ :

$$
\left(\widetilde{S}_{V V}^{(i)}+\widetilde{S}_{V V}^{(j)}+\widetilde{S}_{V V}^{(k)}+\widetilde{S}_{V V}^{(\ell)}\right) \phi=\lambda\left(S_{V V}^{(i)}: S_{V V}^{(j)}: S_{V V}^{(k)}: S_{V V}^{(\ell)}\right) \phi .
$$

This choice replaces the parallel sums in the left-hand side of $V_{\text {par }}$ by a standard sum, motivated by an attempt to reduce the cost of building the generalized eigenproblems.

In order to construct the BDDC primal space, for each of the previous four algorithms, we can select a threshold $0<\theta<1$ and use the following strategy, consisting of two sequential steps:

STEP 1: Select the eigenvectors $\left\{v_{1}, v_{2}, \ldots, v_{N_{c}}\right\}$ of the chosen eigenproblem that are associated with the eigenvalues $\left\{\lambda_{1}, \lambda_{2}, \ldots, \lambda_{N_{c}}\right\}$ that are smaller than $\theta$.

STEP 2: Perform a BDDC change of basis to introduce the selected eigenvectors as new primal constraints.

We now propose two different techniques to perform this change of basis, called in the following AEIG and QR, respectively:

(i) AEIG: This change of basis involves all the eigenvectors of the chosen generalized eigenvalue problem. Therefore, if two different algorithms lead to the same choice of the primal space in STEP 1, the BDDC preconditioners will differ if some of the other eigenvectors differ. The columns of the matrix realizing the BDDC change of basis (see, e.g., [14], [7, eq. (3.16)] for the matrix form of the BDDC preconditioner) are formed by all the eigenvectors.

(ii) QR: This change of basis depends only on the eigenvectors selected in STEP 1 as new primal constraints; thus, in contrast to the AEIG method, it leads to the same BDDC preconditioner when the eigenvectors selected in STEP 1 are the same. The QR method involves the following steps:

1. denoting by $\widetilde{S}_{V} \phi=\lambda S_{V} \phi$ any of the eigenproblems (4.8), (4.9), (4.10), or (4.11), compute the matrix

$$
A_{V}=S_{V}\left[v_{1} v_{2}, \ldots, v_{N_{c}}\right] \in \mathbb{R}^{n \times N_{c}},
$$

with $n$ the size of the $v_{i}, i=1, \ldots, N_{c}$, and $N_{c} \leqslant n$ the number of primal constraints selected;

2. compute the SVD decomposition of $A_{V}$, i.e., the matrices $U, \Sigma, V$ such that

$$
A_{V}=U \Sigma V^{T}
$$

and denote by $C^{T}$ the matrix formed by the first $N_{c}$ columns of $U$; 
3. compute the $\mathrm{QR}$ factorization $C^{T}=Q R$, where

$$
Q=\left[\begin{array}{ll}
Q_{\text {range }} & Q_{\text {null }}
\end{array}\right] \in \mathbb{R}^{n \times n},
$$

with $Q_{\text {range }} \in \mathbb{R}^{n \times N_{c}}$ and $Q_{\text {null }} \in \mathbb{R}^{n \times\left(n-N_{c}\right)}$ spanning the range and the kernel of $C^{T}$, respectively, and

$$
R=\left[\begin{array}{l}
\widetilde{R} \\
0
\end{array}\right] \in \mathbb{R}^{n \times N_{c}},
$$

with $\widetilde{R} \in \mathbb{R}^{N_{c} \times N_{c}}$ upper triangular;

4. construct the matrix $\Phi$ realizing the BDDC change of basis as

$$
\Phi=\left[\begin{array}{ll}
Q_{\text {range }} \widetilde{R}^{-T} & Q_{\text {null }}
\end{array}\right] .
$$

In our 2D tests, we have used the AEIG change of basis, except when otherwise stated, whereas, in our 3D test, only the $\mathrm{QR}$ approach has been employed because the AEIG has not yielded a robust 3D BDDC preconditioner.

5. Numerical results. In our numerical experiments we have worked with the generalized eigenvalue problems $V_{1}(4.8), V_{\text {par }}(4.9), V_{T}$ (4.10), and $V_{m i x}$ (4.11) introduced above and compared the performance of the associated BDDC deluxe preconditioners. The model problem (2.1), with homogeneous Dirichlet boundary conditions, is discretized on a 2D quarter-ring domain and on a 3D twisted domain (see Figure 1) using isogeometric NURBS spaces with a mesh size $h$, polynomial degree $p$, and regularity $k$. The domain is decomposed into $K$ nonoverlapping subdomains of characteristic size $H$, as described in section 3 . The Schur complement problems are solved by the preconditioned conjugate gradient (PCG) method with one of the isogeometric BDDC deluxe preconditioners, with a zero initial guess and and a stopping criterion of a $10^{-6}$ reduction of the Euclidean norm of the PCG residual. In the tests, we study how the convergence rate of the BDDC preconditioner depends on $h, K, p, k$, and jumps in the coefficient of the elliptic problem. In all tests, the BDDC condition number is essentially the maximum eigenvalue of the preconditioned operator, since its minimum eigenvalue is always very close to 1 . The $2 \mathrm{D}$ tests have been performed

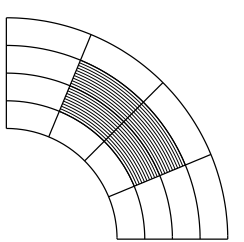

(a) 2D quarter-ring for central jump test.

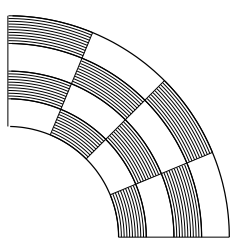

(b) 2D quarter-ring for checkerboard test.

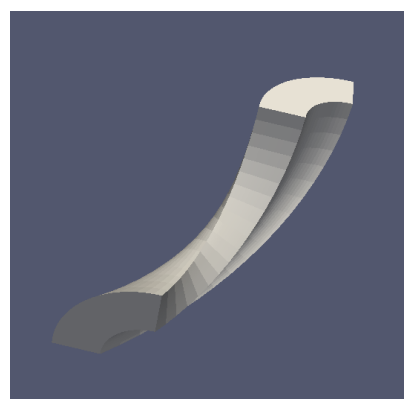

(c) 3D twisted domain.

FIG. 1. Computational domains used in the numerical tests. 

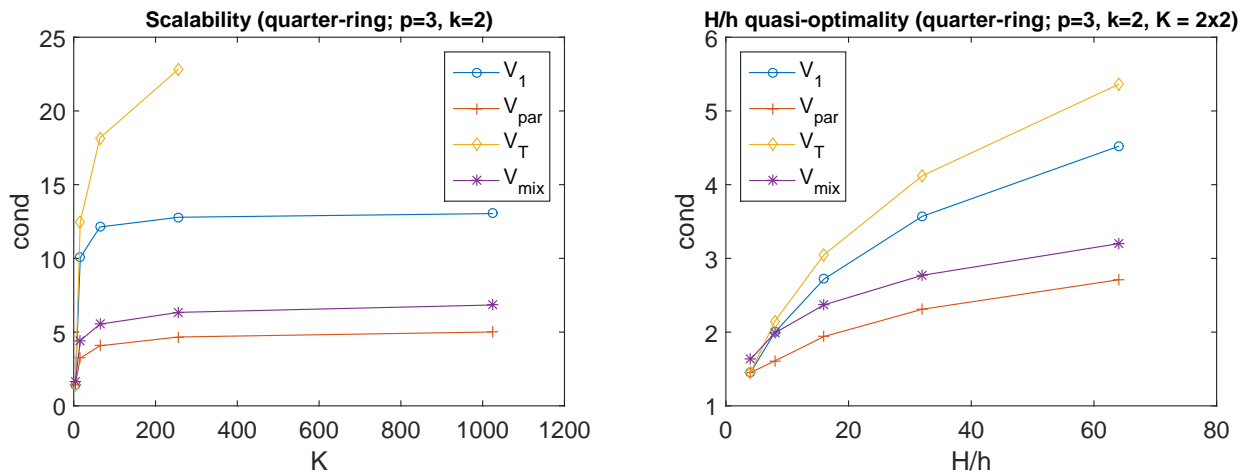

FIG. 2. Performance summary of BDDC deluxe preconditioner with minimal primal spaces $V_{1}$ (o), $V_{\text {par }}(+), V_{T}(\diamond)$, and $V_{\operatorname{mix}}\left(^{*}\right)$. Scability plot (left): Condition number (cond) as a function of the number of subdomains K. Quasi-optimality plot (right): Condition number (cond) as a function of the ratio $H / h$. Quarter-ring domain, $p=3$, and $k=2$.

with a MATLAB code based on the GeoPDEs library [13]. The 3D parallel tests have been performed using the PETSc library [2] and its PCBDDC preconditioner (contributed to the PETSc library by Zampini; see [43]) and run on the parallel machine Shaheen of KAUST (http://www.hpc.kaust.edu.sa/content/shaheen-ii).

In all the following results, we denote by $N_{c}^{V}$ the number of primal variables for each fat vertex, by $N_{c}^{E}$ the number of primal variables for each fat edge, and by $N_{c}^{F}$ the number of primal variables for each fat face. We call a primal space minimal when it includes only one primal variable for each fat object. Thus, the "minimal $V_{1}$ coarse space" uses $N_{c}^{V}=1$ primal variables for each fat vertex, and the "minimal $V E_{1}$ coarse space" employs $N_{c}^{V}=1$ primal variables for each fat vertex and $N_{c}^{E}=1$ primal variables for each fat edge. In some tests, we also explore adaptive primal spaces with more primal vertex variables, i.e., $N_{c}^{V}>1$. We remark that, with the AEIG change of basis, even if the first eigenvector of the generalized vertex eigenproblem turns out to be the same (coinciding often with the average over the fat vertex), irrespective of the kind of eigenproblem considered, the other eigenvectors differ for different choices of primal spaces and give rise to different BDDC changes of basis, and different preconditioners with different performance.

Number of total and primal degrees of freedom (dofs). In the following, we denote by $i V$ the set of interior subdomain vertices, by $i E$ the set of interior subdomain edges, and by $i F$ the set of interior subdomain faces.

Then in the $2 \mathrm{D}$ tests, we have

- \# total dofs $=(1 / h+p-2)^{2}$,

- \# primal dofs $=N_{c}^{V}(\# i V)+N_{c}^{E}(\# i E)$,

and in 3D tests, we have

- \# total dofs $=(1 / h+p-2)^{3}$,

- \# primal dofs $=N_{c}^{V}(\# i V)+N_{c}^{E}(\# i E)+N_{c}^{F}(\# i F)$.

5.1. 2D results. Before considering the results for each primal choice in detail, we note, in summary, that our numerical results indicate that all primal choices considered are scalable in the number of subdomains $K$ and quasi-optimal in the ratio $H / h$; see Figure 2 (two plots in the left panel have fewer points because their generalized eigenproblem broke down for the larger meshes). The performance with 
TABLE 1

$B D D C$ deluxe preconditioner with the minimal $V_{1}$ coarse space for a quarter-ring domain: condition number, cond, and iteration counts, $\mathrm{n}_{\mathrm{it}}$, as functions of (a) the number of subdomains $K$ and mesh size $h$ for $p=3, k=2$; (b) the polynomial degree $p$ for fixed $K=4 \times 4, H / h=16$ (* means that the preconditioner breaks down; see text). (c) Condition number of the unpreconditioned IGA stiffness matrix $A$ and its Schur complement $\widehat{S}$ as functions of the polynomial degree $p$ for B-splines on a square domain with mesh size $1 / h=64$, number of subdomains $K=4 \times 4$, maximal regularity $k=p-1$, and regularity at the subdomain interface $k_{\Gamma}=1$.

(a)

\begin{tabular}{c|rr|rr|rr|rr|rr}
\hline & \multicolumn{2}{|c|}{$h=1 / 8$} & \multicolumn{2}{|c|}{$h=1 / 16$} & \multicolumn{2}{|c|}{$h=1 / 32$} & \multicolumn{2}{|c|}{$h=1 / 64$} & \multicolumn{2}{c}{$h=1 / 128$} \\
$K$ & cond & $\mathrm{n}_{\text {it }}$ & cond & $\mathrm{n}_{\text {it }}$ & cond & $\mathrm{n}_{\text {it }}$ & cond & $\mathrm{n}_{\text {it }}$ & cond & $\mathrm{n}_{\text {it }}$ \\
\hline $2 \times 2$ & 1.45 & 7 & 2.00 & 8 & 2.72 & 8 & 3.57 & 8 & 4.52 & 8 \\
$4 \times 4$ & & & 10.06 & 15 & 13.90 & 16 & 18.66 & 18 & 23.92 & 21 \\
$8 \times 8$ & & & & & 12.13 & 24 & 17.42 & 27 & 24.85 & 32 \\
$16 \times 16$ & & & & & & & 12.79 & 24 & 18.96 & 29 \\
$32 \times 32$ & & & & & & & & & 13.04 & 24 \\
\hline
\end{tabular}

\begin{tabular}{|c|c|c|c|c|c|c|}
\hline \multicolumn{7}{|c|}{ (b) } \\
\hline \multirow[b]{2}{*}{$p$} & \multicolumn{2}{|c|}{$k=p-1$} & \multicolumn{2}{|c|}{$k=2$} & \multicolumn{2}{|c|}{$k=1$} \\
\hline & cond & $\mathrm{n}_{\mathrm{it}}$ & cond & $\mathrm{n}_{\mathrm{it}}$ & cond & $\mathrm{n}_{\text {it }}$ \\
\hline 2 & 7.09 & 14 & $\mathrm{n} /$ & & 7.09 & 14 \\
\hline 3 & 18.66 & 18 & 18.66 & 18 & 7.59 & 15 \\
\hline 4 & 233.81 & 26 & 19.74 & 20 & 8.31 & 15 \\
\hline 5 & $8.42 \mathrm{e} 3$ & 56 & 22.22 & 19 & 9.06 & 15 \\
\hline 6 & * & & 25.37 & 21 & 9.81 & 16 \\
\hline 7 & * & & 29.05 & 22 & 10.52 & 16 \\
\hline 8 & * & & 33.08 & 23 & 11.24 & 17 \\
\hline 9 & * & & 37.64 & 24 & 11.90 & 17 \\
\hline 10 & * & & 39.89 & 26 & 12.59 & 18 \\
\hline
\end{tabular}

\begin{tabular}{r|r|r}
\multicolumn{3}{c}{$(\mathrm{c})$} \\
\hline$p$ & $\begin{array}{r}\text { Unprec. } \\
\text { cond }(A)\end{array}$ & $\begin{array}{c}\text { Schur } \\
\text { cond }(\widehat{S})\end{array}$ \\
\hline 2 & 311.46 & 72.57 \\
3 & 366.81 & 75.99 \\
4 & 477.38 & 90.27 \\
5 & $1.78 \mathrm{e} 3$ & 114.56 \\
6 & $1.49 \mathrm{e} 4$ & 338.43 \\
7 & $1.27 \mathrm{e} 5$ & $1.00 \mathrm{e} 3$ \\
8 & $1.11 \mathrm{e} 6$ & $2.98 \mathrm{e} 3$ \\
9 & $1.01 \mathrm{e} 7$ & $8.77 \mathrm{e} 3$ \\
10 & $9.42 \mathrm{e} 7$ & $2.56 \mathrm{e} 4$ \\
\hline
\end{tabular}

respect to the polynomial degree $p$ degenerates in case of maximal regularity $k=p-1$ for the primal choices $V_{1}$ and $V_{T}$ with the minimal primal spaces with $N_{c}^{V}=1$, but good performance can be recovered by the minimal coarse space using richer primal spaces with $N_{c}^{V}>1$. We do not consider richer primal choices for $V_{\text {par }}$ and $V_{m i x}$ since the minimal coarse space for each fat vertex already yields good performance in $p$ independently of the regularity $k$. Some tests marked by $*$ in the following tables could not be run since either the MATLAB generalized eigensolver broke down due to the extreme ill-conditioning of the Schur complement involved, returning spurious complex eigenvalues and eigenvectors, or the local problems after the BDDC change of basis were almost singular, causing the local solvers to break down.

Minimal $V_{1}$ primal space $\left(N_{c}^{V}=1\right)$. Table 1 reports on the BDDC condition numbers and iteration counts with the minimal $V_{1}$ primal space using only one primal constraint for each fat vertex $\left(N_{c}^{V}=1\right)$ for a quarter-ring domain. Table 1(a) shows the results for varying the number of subdomains $K$ and the fine mesh size $h$ for fixed $p=3, k=2$. Moving along the diagonal of the table, the subdomain to element mesh size ratio $H / h$ remains constant, and we see that this primal choice is scalable for an increasing number of subdomains $K$ and is quasi-optimal with respect to the ratio $H / h$. We have also plotted the condition numbers in Figure 2 for greater clarity. Table 1(b) reports the BDDC condition numbers and iteration counts for an increasing polynomial degree up to $p=10$ for low regularity $k=1$ (right column), $k=2$ (middle column), and maximal regularity $k=p-1$ (left column) for fixed $K=4 \times 4, H / h=16$. We see that the minimal $V_{1}$ primal space performs well for $k=1$ and 2 but very poorly for $k=p-1$, since the condition numbers seem to 
TABLE 2

$B D D C$ deluxe preconditioner with the minimal $V E_{1}$ coarse space for a quarter-ring domain: Condition number, cond, and iteration counts, $\mathrm{n}_{\mathrm{it}}$, as functions of (a) the number of subdomains $K$ and mesh size $h$ for fixed $p=3, k=2$; (b) the polynomial degree $p$ for fixed $K=4 \times 4, H / h=16$ (* means that the preconditioner breaks down; see text).

\begin{tabular}{c|rr|rr|rr|rr|rr}
\multicolumn{10}{c}{$(\mathrm{a})$} \\
\hline & \multicolumn{2}{|c|}{$h=1 / 8$} & \multicolumn{2}{c|}{$h=1 / 16$} & \multicolumn{2}{c|}{$h=1 / 32$} & \multicolumn{2}{c|}{$h=1 / 64$} & \multicolumn{2}{c}{$h=1 / 128$} \\
$K$ & cond & $\mathrm{n}_{\text {it }}$ & cond & $\mathrm{n}_{\text {it }}$ & cond & $\mathrm{n}_{\text {it }}$ & cond & $\mathrm{n}_{\text {it }}$ & cond & $\mathrm{n}_{\text {it }}$ \\
\hline $2 \times 2$ & 1.44 & 7 & 1.97 & 7 & 2.65 & 8 & 3.46 & 8 & 4.37 & 8 \\
$4 \times 4$ & & & 5.09 & 13 & 4.65 & 13 & 5.31 & 14 & 5.99 & 15 \\
$8 \times 8$ & & & & & 6.20 & 17 & 5.34 & 15 & 6.00 & 16 \\
$16 \times 16$ & & & & & & & 6.66 & 18 & 5.73 & 16 \\
$32 \times 32$ & & & & & & & & & 6.83 & 18 \\
\hline
\end{tabular}

\begin{tabular}{|c|c|c|c|c|c|c|}
\hline \multirow[b]{3}{*}{$p$} & \multicolumn{6}{|c|}{ (b) } \\
\hline & \multicolumn{2}{|c|}{$k=p-1$} & \multicolumn{2}{|c|}{$k=2$} & \multicolumn{2}{|c|}{$k=1$} \\
\hline & cond & $\mathrm{n}_{\mathrm{it}}$ & cond & $n_{i t}$ & cond & $n_{i t}$ \\
\hline 2 & 2.91 & 11 & $n$ & & 2.91 & 11 \\
\hline 3 & 5.31 & 14 & 5.31 & 14 & 2.80 & 11 \\
\hline 4 & 41.17 & 24 & 4.85 & 21 & 2.88 & 11 \\
\hline 5 & 1598.65 & 67 & 4.77 & 14 & 3.00 & 11 \\
\hline 6 & * & & 4.93 & 15 & 3.13 & 11 \\
\hline 7 & * & & 5.16 & 16 & 3.27 & 12 \\
\hline 8 & * & & 5.67 & 17 & 3.40 & 12 \\
\hline 9 & * & & * & & 3.53 & 13 \\
\hline 10 & * & & * & & 3.71 & 13 \\
\hline
\end{tabular}

grow exponentially in $p$ and the generalized eigenproblem/change of basis $V_{1}$ breaks down for $p \geqslant 6$. We remark that the unpreconditioned discrete problem is very illconditioned: Table 1(c) reports the condition number of the unpreconditioned IGA stiffness matrix $A$ and its Schur complement $\widehat{S}$ as functions of the polynomial degree $p$ for B-splines on a square domain with mesh size $1 / h=64$, number of subdomains $K=4 \times 4$, maximal regularity $k=p-1$, and regularity at the subdomain interface $k_{\Gamma}=1$. Additional $2 \mathrm{D}$ and $3 \mathrm{D}$ results for unpreconditioned IGA problems can be found in our previous paper [5].

Minimal $V E_{1}$ primal space $\left(N_{c}^{V}=1, N_{c}^{E}=1\right)$. Table 2 reports analogous tests for the minimal $V E_{1}$ primal space where edge primal constraints are added to the vertex ones $\left(N_{c}^{V}=1, N_{c}^{E}=1\right)$. In Table $2($ a), we now consider only the classical $p=3, k=2$ case. As expected, adding the edge constraints to the primal space improves all the results (see the corresponding results of the previous Table 1). The method remains scalable in $K$, quasi-optimal in $H / h$, and performs well for increasing $p$ and low regularity $k=1$ and 2, but still degenerates for increasing $p$ for $k=p-1$.

Adaptive $V_{1}$ primal space. In Table 3 , we then study the adaptive primal space $V_{1}$ on a square domain to see the effect of adding more vertex primal constraints in addition to the minimal choice of just one. We consider both the minimal choice of $N_{c}^{V}=1$ primal vertex constraint and a richer choice of $N_{c}^{V}=4$ primal vertex constraints for each subdomain vertex. In case of increasing polynomial degree $p$, we also consider a lower threshold $\theta=10 / 11$ that leads to a richer choice of approximately $N_{c}^{V}=2 p$ primal constraints for each subdomain vertex. The results in (a) show that this BDDC deluxe preconditioner is scalable, since cond and $n_{\text {it }}$ appear to be bounded from above by a constant independent of $K$, and the results in (b) indicate that the preconditioner is quasi-optimal, since cond and $n_{i t}$ appear to grow polylogarithmically 
TABLE 3

$B D D C$ deluxe preconditioner with an adaptive choice of the $V_{1}$ coarse space on a square domain, with $N_{c}^{V}$ selected primal constraints for each fat vertex. Condition number cond and iteration counts $\mathrm{n}_{\mathrm{it}}$ as functions of (a) the number of subdomains $K$ for fixed $p=3, k=2, H / h=8$; (b) the ratio $H / h$ for fixed $p=3, k=2, K=4 \times 4$; (c) the polynomial degree $p$ for fixed $K=4 \times 4, H / h=16$, $k=p-1$ (* means that the preconditioner breaks down; see text).

(a)

\begin{tabular}{c|rr|rr}
\hline & \multicolumn{2}{|c|}{$N_{c}^{V}=1$} & \multicolumn{2}{c}{$N_{c}^{V}=4$} \\
$K$ & cond & $\mathrm{n}_{\text {it }}$ & cond & $\mathrm{n}_{\text {it }}$ \\
\hline $2 \times 2$ & 1.81 & 7 & 1.66 & 8 \\
$4 \times 4$ & 12.74 & 14 & 6.74 & 13 \\
$8 \times 8$ & 14.74 & 24 & 7.48 & 18 \\
$16 \times 16$ & 15.67 & 26 & 7.78 & 18 \\
$32 \times 32$ & 16.13 & 24 & 7.87 & 17 \\
\hline
\end{tabular}

(b)

\begin{tabular}{r|rr|rr}
\multicolumn{9}{c}{$(\mathrm{b})$} \\
\hline
\end{tabular}

(c)

\begin{tabular}{c|rr|rrr}
\hline & \multicolumn{2}{|c|}{$N_{c}^{V}=1$} & & & \\
$p$ & cond & $\mathrm{n}_{\text {it }}$ & cond & $\mathrm{n}_{\text {it }}$ & $N_{c}^{V}$ \\
\hline 2 & 6.09 & 13 & 3.55 & 11 & 3 \\
3 & 17.40 & 17 & 5.34 & 14 & 5 \\
4 & 230.9 & 21 & 5.74 & 15 & 8 \\
5 & 7545.9 & 39 & 12.25 & 18 & 10 \\
6 & $*$ & & 73.08 & 31 & 12 \\
\hline
\end{tabular}

TABLE 4

$B D D C$ deluxe preconditioner with the minimal $V_{\text {par }}$ coarse space for a quarter-ring domain: Condition number, cond, and iteration counts, $\mathrm{n}_{\mathrm{it}}$, as functions of (a) the number of subdomains $K$ and mesh size $h$ for fixed $p=3, k=2$; (b) the polynomial degree $p$ for fixed $K=4 \times 4, H / h=16$ (* means that the change of basis is performed by the QR approach).

\begin{tabular}{c|rr|rr|rr|rr|rr}
\multicolumn{10}{c|}{$(\mathrm{a})$} \\
\hline & \multicolumn{2}{|c|}{$h=1 / 8$} & \multicolumn{2}{c|}{$h=1 / 16$} & \multicolumn{2}{c|}{$h=1 / 32$} & \multicolumn{2}{c|}{$h=1 / 64$} & \multicolumn{2}{c}{$h=1 / 128$} \\
$K$ & cond & $\mathrm{n}_{\text {it }}$ & cond & $\mathrm{n}_{\text {it }}$ & cond & $\mathrm{n}_{\text {it }}$ & cond & $\mathrm{n}_{\text {it }}$ & cond & $\mathrm{n}_{\text {it }}$ \\
\hline $2 \times 2$ & 1.45 & 7 & 1.61 & 7 & 1.94 & 7 & 2.31 & 8 & 2.71 & 8 \\
$4 \times 4$ & & & 3.24 & 11 & 4.19 & 12 & 5.20 & 13 & 6.32 & 13 \\
$8 \times 8$ & & & & & 4.07 & 13 & 5.36 & 12 & 6.77 & 17 \\
$16 \times 16$ & & & & & & & 4.67 & 15 & 6.21 & 16 \\
$32 \times 32$ & & & & & & & & & $* 5.01$ & 15 \\
\hline
\end{tabular}

\begin{tabular}{|c|c|c|c|c|c|c|}
\hline \multirow[b]{3}{*}{$p$} & \multicolumn{6}{|c|}{ (b) } \\
\hline & \multicolumn{2}{|c|}{$k=p-1$} & \multicolumn{2}{|c|}{$k=2$} & \multicolumn{2}{|c|}{$k=1$} \\
\hline & cond & $n_{i t}$ & cond & $\mathrm{n}_{\mathrm{it}}$ & cond & $\mathrm{n}_{\mathrm{it}}$ \\
\hline 2 & 5.54 & 13 & \multicolumn{2}{|c|}{$\mathrm{n} / \mathrm{a}$} & 5.54 & 13 \\
\hline 3 & 5.20 & 13 & 5.20 & 13 & 6.01 & 13 \\
\hline 4 & 6.02 & 14 & 5.54 & 13 & 6.47 & 13 \\
\hline 5 & 5.77 & 14 & 5.96 & 14 & 6.89 & 14 \\
\hline 6 & 6.35 & 16 & 6.39 & 14 & 7.27 & 14 \\
\hline 7 & *6.32 & 16 & 6.86 & 16 & 7.62 & 15 \\
\hline 8 & *9.34 & 21 & 7.36 & 17 & 7.93 & 15 \\
\hline 9 & *21.6 & 31 & 8.04 & 20 & 8.23 & 16 \\
\hline 10 & *53.7 & 61 & 9.26 & 22 & 8.50 & 17 \\
\hline
\end{tabular}

in $H / h$. The results in (c) confirm that the minimal choice $N_{c}^{V}=1$ does not perform well for an increasing $p$, while with the richer choice with increasing number of vertex primal variables, we observe only a mild performance degradation for $p \leqslant 6$.

Minimal $V_{\text {par }}$ primal space. Table 4 shows that the results improve considerably with the minimal $V_{\text {par }}$ primal space for a quarter-ring domain. Again we observe scalability and quasi-optimality in Table 4(a) (see also Figure 2) and a good performance

Copyright $@$ ( ) by SIAM. Unauthorized reproduction of this article is prohibited. 
TABLE 5

$B D D C$ deluxe preconditioner with the minimal and adaptive choice of $V_{T}$ coarse space for a quarter-ring domain, with $N_{c}^{V}$ primal constraints for each fat vertex. Condition number, cond, and iteration counts, $\mathrm{n}_{\mathrm{it}}$, as functions of (a) the number of subdomains $K$ and mesh size $h$ for fixed $p=3, k=2$ and the minimal $N_{c}^{V}=1$; (b) the polynomial degree $p$ for fixed $K=4 \times 4, H / h=16$ and both the minimal and adaptive of $N_{c}^{V}$ ( ${ }^{*}$ means that the preconditioner breaks down; see text).

(a)

\begin{tabular}{c|rr|rr|rr|rr|rr}
\hline & \multicolumn{2}{|c|}{$h=1 / 8$} & \multicolumn{2}{|c|}{$h=1 / 16$} & \multicolumn{2}{|c|}{$h=1 / 32$} & \multicolumn{2}{c|}{$h=1 / 64$} & \multicolumn{2}{c}{$h=1 / 128$} \\
$K$ & cond & $\mathrm{n}_{\text {it }}$ & cond & $\mathrm{n}_{\text {it }}$ & cond & $\mathrm{n}_{\text {it }}$ & cond & $\mathrm{n}_{\text {it }}$ & cond & $\mathrm{n}_{\text {it }}$ \\
\hline $2 \times 2$ & 1.45 & 7 & 2.15 & 7 & 3.05 & 8 & 4.12 & 8 & 5.36 & 8 \\
$4 \times 4$ & & & 12.49 & 14 & 16.18 & 16 & 19.74 & 17 & 23.32 & 17 \\
$8 \times 8$ & & & & & 18.17 & 24 & 21.81 & 26 & 26.39 & 28 \\
$16 \times 16$ & & & & & & & 22.83 & 30 & 26.09 & 31 \\
$32 \times 32$ & & & & & & & & & $*$ & \\
\hline
\end{tabular}

(b)

\begin{tabular}{|c|c|c|c|c|c|c|c|c|c|}
\hline \multirow[b]{3}{*}{$p$} & \multicolumn{6}{|c|}{$N_{c}^{V}=1$} & \multirow{2}{*}{\multicolumn{3}{|c|}{$\begin{array}{c}\text { Adaptive } N_{c}^{V} \\
k=p-1\end{array}$}} \\
\hline & \multicolumn{2}{|c|}{$k=p-1$} & \multicolumn{2}{|c|}{$k=2$} & \multicolumn{2}{|c|}{$k=1$} & & & \\
\hline & cond & $\mathrm{n}_{\mathrm{it}}$ & cond & $\mathrm{n}_{\mathrm{it}}$ & cond & $\mathrm{n}_{\mathrm{it}}$ & cond & $\mathrm{n}_{\mathrm{it}}$ & $N_{c}^{V}$ \\
\hline 2 & 5.53 & 13 & $\mathrm{n} /$ & & 5.53 & 13 & 4.33 & 12 & 3 \\
\hline 3 & 19.74 & 17 & 19.74 & 17 & 6.00 & 13 & 9.09 & 15 & 5 \\
\hline 4 & 499.95 & 24 & 20.92 & 16 & 6.46 & 13 & 10.62 & 14 & 10 \\
\hline 5 & $1.65 \mathrm{e}+4$ & 31 & 23.03 & 19 & 6.89 & 14 & 10.62 & 15 & 15 \\
\hline 6 & $*$ & & 25.57 & 18 & 7.27 & 14 & 9.39 & 17 & 25 \\
\hline 7 & * & & 28.50 & 21 & 7.61 & 15 & 21.95 & 20 & 34 \\
\hline 8 & * & & 31.48 & 22 & 7.93 & 15 & * & & \\
\hline
\end{tabular}

in $p$ in Table 4(b) even in case of maximal regularity $k=p-1$ up to $p=8$. Note that in case of maximal regularity for $p \geqslant 7$, the AEIG generalized eigenproblems/change of basis break down, while the QR change of basis allows us to reach convergence. Figure 2 shows that the minimal $V_{\text {par }}$ primal space yields the best performance among the choices of primal constraints that we have considered.

Minimal and adaptive $V_{T}$ primal space. The results of Table 5 show that the minimal $V_{T}$ primal space for a quarter-ring domain is still scalable in $K$ and quasioptimal in $H / h$ (Table $5($ a) and Figure 2 ) but with slightly larger condition numbers and iteration counts than the previous primal choices. The performance in $p$ in Table $5(\mathrm{~b})$ for maximal regularity $k=p-1$ using only the minimal $V_{T}$ primal space (left columns) degenerates again with increasing $p$ as for the minimal $V_{1}$ and $V E_{1}$ primal spaces, with an analogous breakdown for $p=6$, but good performance can be recovered for low regularity $k=2$ and 1 (middle columns). A good performance can also be recovered in the maximal regularity case $k=p-1$ by adaptively increasing the number $N_{c}^{V}$ of vertex primal constraints to about $70 \%$ of the total number, $p^{2}$, of possible vertex constraints for each fat vertex (last columns).

Minimal $V_{m i x}$ primal space. Table 6 shows that the minimal $V_{m i x}$ primal space for a quarter-ring domain yields the second-best performance among the choices of primal constraints considered. Indeed, Table 6 (a) shows scalability and quasi optimality with quite small condition numbers and iteration counts; see also Figure 2. Table 6(b) reports much better results than the previous coarse space choices for increasing $p$, even in case of maximal regularity $k=p-1$ (left column) up to $p=8$, with the eigenproblem/change of basis breakdown occurring only for $p \geqslant 9$.

Robustness with respect to jumping coefficients. We also study the robustness of the BDDC deluxe preconditioner with respect to jump discontinuities of the coefficient of the elliptic problem $\rho$. Due to space limitation, we report the results only for the 
TABLE 6

$B D D C$ deluxe preconditioner with the minimal $V_{m i x}$ coarse space for a quarter-ring domain: Condition number, cond, and iteration counts, $\mathrm{n}_{\mathrm{it}}$, as functions of (a) the number of subdomains $K$ and mesh size $h$ for fixed $p=3, k=2$; (b) the polynomial degree $p$, for fixed $K=4 \times 4, H / h=16$ ( ${ }^{*}$ means that the preconditioner breaks down; see text).

\begin{tabular}{c|rr|rr|rr|rr|rr}
\multicolumn{10}{c|}{$h=1 / 32$} & \multicolumn{2}{|c|}{$h=1 / 64$} & \multicolumn{2}{c}{$h=1 / 128$} \\
\hline & \multicolumn{2}{|c|}{$h=1 / 8$} & \multicolumn{2}{c|}{$h=1 / 16$} & \multicolumn{2}{c|}{$h=1$} \\
$K$ & cond & $\mathrm{n}_{\text {it }}$ & cond & $\mathrm{n}_{\text {it }}$ & cond & $\mathrm{n}_{\text {it }}$ & cond & $\mathrm{n}_{\text {it }}$ & cond & $\mathrm{n}_{\text {it }}$ \\
\hline $2 \times 2$ & 1.63 & 7 & 1.99 & 7 & 2.37 & 8 & 2.77 & 8 & 3.20 & 8 \\
$4 \times 4$ & & & 4.43 & 12 & 5.43 & 13 & 6.53 & 14 & 7.74 & 15 \\
$8 \times 8$ & & & & & 5.54 & 15 & 6.91 & 17 & 8.33 & 18 \\
$16 \times 16$ & & & & & & & 6.34 & 17 & 7.99 & 19 \\
$32 \times 32$ & & & & & & & & 6.84 & 17 \\
\hline
\end{tabular}

\begin{tabular}{|c|c|c|c|c|c|c|}
\hline \multicolumn{7}{|c|}{ (b) } \\
\hline \multirow[b]{2}{*}{$p$} & \multicolumn{2}{|c|}{$k=p-1$} & \multicolumn{2}{|c|}{$k=2$} & \multicolumn{2}{|c|}{$k=1$} \\
\hline & cond & $\mathrm{n}_{\mathrm{it}}$ & cond & $\mathrm{n}_{\mathrm{it}}$ & cond & $\mathrm{n}_{\mathrm{it}}$ \\
\hline 2 & 5.39 & 13 & $\mathrm{n} /$ & & 5.39 & 13 \\
\hline 3 & 5.43 & 13 & 5.43 & 13 & 6.01 & 13 \\
\hline 4 & 5.91 & 13 & 5.76 & 13 & 6.47 & 13 \\
\hline 5 & 6.13 & 14 & 6.41 & 14 & 6.89 & 14 \\
\hline 6 & 6.27 & 14 & 7.20 & 14 & 7.27 & 14 \\
\hline 7 & 7.29 & 18 & 7.33 & 16 & 7.62 & 15 \\
\hline 8 & 9.33 & 24 & 7.36 & 17 & 11.41 & 16 \\
\hline 9 & * & & 8.33 & 20 & 8.23 & 16 \\
\hline 10 & $*$ & & 9.47 & 22 & 8.50 & 17 \\
\hline
\end{tabular}

minimal $V_{T}$ and $V_{\text {par }}$ primal choices and three different classical tests, which we call "central jump," "checkerboard," and "random mix" for a 2D quarter-ring domain decomposed into $4 \times 4$ subdomains; cf. also [7]. In the central jump test (see Figure $1(\mathrm{a})$ ), the coefficient $\rho$ varies by 8 orders of magnitude (from $10^{-4}$ to $10^{4}$ ) in the $2 \times 2$ central subdomains, while it equals 1 in the surrounding subdomains. In the checkerboard test (see Figure 1(b)), $\rho$ is $10^{4}$ or $10^{-4}$ in the white subdomains and 1 in the black subdomains. In the random mix test, $\rho$ has random values varying by 8 orders of magnitude between the different subdomains. We fix $h=1 / 64, H / h=16$, and we test different splines spaces: $p=2, k=1 ; p=3, k=1 ; p=3, k=2$. Tables 7 and 8 report the condition number of the preconditioned system and the conjugate gradient iteration counts. The results show clearly the robustness of BDDC, for both choices of primal spaces.

5.2. 3D results. In Figure 3, we report results of parallel numerical experiments on a 3D NURBS domain shown in Figure 1(c) and using the PCBDDC PETSc objects (see [43]) to implement BDDC deluxe with the $V E F_{\text {par }}$ coarse space, i.e., with primal constraints for vertices $(\mathrm{V})$, edges $(\mathrm{E})$, and faces $(\mathrm{F})$. We study only this primal choice because $V_{\text {par }}$ was the algorithm attaining the best performance in our $2 \mathrm{D}$ results. Let us denote by $N_{c}$ the maximum number of constraints among all equivalence classes, i.e., $N_{c}=\max \left(N_{c}^{V}, N_{c}^{E}, N_{c}^{F}\right)$. The coarse space is minimal, i.e., $N_{c}=1$, for the scalability test in panel (b) and the quasi-optimality test in panel (c), while it is both minimal and adaptive, i.e., $N_{c} \geqslant 1$ in panel (d). The number of processors used in each test equals the number of subdomains $K$. The scalability test (b) shows that condition numbers and iteration counts are bounded from above when the number of subdomains $K$ is increased for fixed $p=3, k=2, H / h=6$. The results of the quasioptimality test (c) are less clear, initially showing almost constant condition numbers and iteration counts for $6 \leqslant H / h \leqslant 9$ and then a modest growth up to $H / h=12$ (the 
TABLE 7

Robustness with respect to jumping coefficients: Central jump, checkerboard, and random mix tests for $B D D C$ deluxe preconditioner with the minimal $V_{T}$ coarse space for a quarter-ring domain. The reported quantities are the condition number, cond, of the preconditioned system and the conjugate gradient iteration counts, $\mathrm{n}_{\mathrm{it}}$. The spline spaces considered are $p=2, k=1 ; p=3, k=1$; $p=3, k=2$. Fixed mesh size $h=1 / 64$, number of subdomains $K=4 \times 4$, subdomain size $H / h=16$.

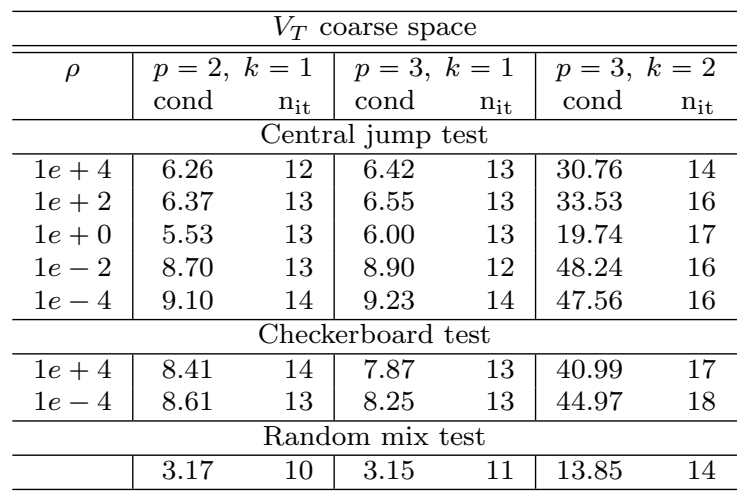

TABLE 8

Robustness with respect to jumping coefficients: central jump, checkerboard and random mix tests for $B D D C$ deluxe preconditioner with the minimal $V_{\text {par }}$ coarse space in a quarter-ring domain. Same format as in Table 7.

\begin{tabular}{c|rr|rr|rr}
\hline \multicolumn{7}{c}{$V_{\text {par }}$ coarse space } \\
\hline \hline$\rho$ & $\begin{array}{r}p=2, \\
\text { cond }\end{array}$ & $k=1$ & \multicolumn{2}{c}{$p=3, k=1$} & $p=3, k=2$ \\
& $\mathrm{n}_{\text {it }}$ & cond & $\mathrm{n}_{\text {it }}$ & cond & $\mathrm{n}_{\text {it }}$ \\
\hline \multicolumn{7}{c}{ Central jump test } \\
\hline $1 e+4$ & 10.63 & 12 & 11.40 & 12 & 16.38 & 13 \\
$1 e+2$ & 10.31 & 13 & 11.06 & 13 & 14.56 & 13 \\
$1 e+0$ & 5.54 & 13 & 6.01 & 13 & 5.20 & 13 \\
$1 e-2$ & 4.82 & 14 & 4.89 & 14 & 7.03 & 15 \\
$1 e-4$ & 4.90 & 15 & 4.94 & 14 & 7.54 & 16 \\
\hline \multicolumn{7}{c}{ Checkerboard test } \\
\hline $1 e+4$ & 7.75 & 13 & 8.35 & 14 & 33.59 & 15 \\
$1 e-4$ & 7.08 & 13 & 7.60 & 14 & 27.75 & 16 \\
\hline \multicolumn{7}{c}{ Random mix test } \\
\hline & 5.28 & 12 & 5.65 & 13 & 22.19 & 17 \\
\hline
\end{tabular}

other parameters are fixed at $p=3, k=2, K=4 \times 4 \times 4$ ). The last test (d) for increasing polynomial degree $p$ (and fixed $K=4 \times 4 \times 4, H / h=8, k=p-1$ ) shows, besides an unexpected decrease of the condition numbers when increasing $p$ from 2 to 3 , a more than linear growth for $p \geqslant 3$ with the minimal choice $N_{c}=1$. This growth can be considerably improved by adaptively increasing $N_{c} \geqslant 1$ as shown in the table.

6. Conclusions. We have developed several algorithms for the adaptive selection of primal constraints in BDDC deluxe preconditioners applied to isogeometric discretizations of scalar elliptic problems. These new algorithms allow us to significantly reduce the coarse space dimensions compared with those of previously developed BDDC isogeometric preconditioners. For one of the proposed algorithms, the $V_{T}$ choice, we have derived a theoretical estimate implying the quasi optimality and scalability of the associated BDDC preconditioner. Two- and three-dimensional numerical tests demonstrate the quasi optimality, scalability, and robustness of our algorithms 
(a) Minimal $\mathcal{V E F}_{\text {par }}$.

\begin{tabular}{c|rr}
\hline$K$ & cond & $\mathrm{n}_{\text {it }}$ \\
\hline $2 \times 2 \times 2$ & 2.2 & 8 \\
$3 \times 3 \times 3$ & 10.1 & 16 \\
$4 \times 4 \times 4$ & 13.4 & 22 \\
$5 \times 5 \times 5$ & 15.4 & 24 \\
$6 \times 6 \times 6$ & 16.8 & 25 \\
$7 \times 7 \times 7$ & 17.8 & 26 \\
$8 \times 8 \times 8$ & 18.5 & 26 \\
$9 \times 9 \times 9$ & 19.8 & 27 \\
$10 \times 10 \times 10$ & 19.6 & 27 \\
\hline
\end{tabular}

(b) Minimal $\mathcal{V E F}_{\text {par }}$.

\begin{tabular}{c|cc}
\hline$H / h$ & cond & $\mathrm{n}_{\text {it }}$ \\
\hline 6 & 13.4 & 22 \\
7 & 12.8 & 21 \\
8 & 12.8 & 21 \\
9 & 12.9 & 21 \\
10 & 13.1 & 21 \\
11 & 13.3 & 22 \\
12 & 13.6 & 22 \\
\hline
\end{tabular}

(c) Minimal and adaptive $\mathcal{V E F}_{\text {par }}$.

\begin{tabular}{r|rrr|rrr|rrr}
\hline & \multicolumn{7}{|c|}{ Minimal } & \multicolumn{7}{c}{ Adaptive } \\
$p$ & cond & $\mathrm{n}_{\text {it }}$ & $N_{c}$ & cond & $\mathrm{n}_{\text {it }}$ & $N_{c}$ & cond & $\mathrm{n}_{\text {it }}$ & $N_{c}$ \\
\hline 2 & 31.9 & 25 & 1 & 31.8 & 24 & 1 & 17.4 & 19 & 2 \\
3 & 12.8 & 21 & 1 & 12.8 & 21 & 1 & 11.5 & 20 & 2 \\
4 & 19.2 & 23 & 1 & 14.7 & 22 & 4 & 14.2 & 21 & 16 \\
5 & 44.1 & 32 & 1 & 21.0 & 26 & 18 & 15.3 & 22 & 40 \\
\hline
\end{tabular}

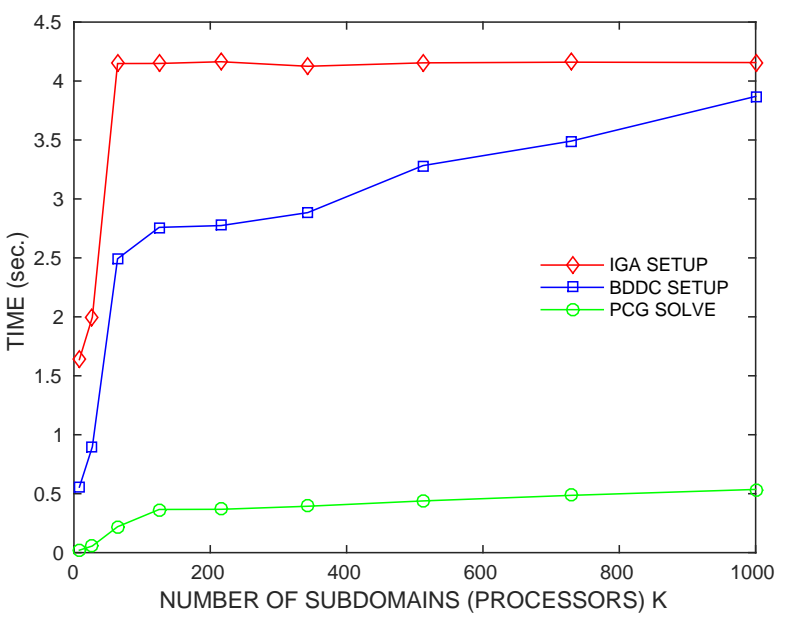

(d) Parallel timings for the scalability test of table (a).

FIG. 3. 3D parallel tests with BDDC deluxe preconditioner with $V E F_{\text {par }}$ coarse space on a $3 D$ NURB domain shown in Figure 1(c). Number of processors $=$ number of subdomains $K$. Condition number cond and iteration counts $\mathrm{n}_{\mathrm{it}}$ as functions of (a) the number of subdomains $K$ for fixed $p=3, k=2, H / h=6$; (b) the ratio $H / h$ for fixed $p=3, k=2, K=4 \times 4 \times 4$; (c) the polynomial degree $p$ for fixed $K=4 \times 4 \times 4, H / h=8, k=p-1$, with both the minimal and adaptive choices of primal constraints $\left(N_{c}=\max \left(N_{c}^{V}, N_{c}^{E}, N_{c}^{F}\right)\right.$ is the maximum number of primal constraints for each equivalence class); (d) Parallel timings for the scalability test of table (a).

with respect to the spline polynomial degree and the presence of discontinuous elliptic coefficients. The numerical results have shown that the most effective primal spaces for our deluxe BDDC algorithms are $V_{\text {par }}$ and $V_{m i x}$, particularly for isogeometric discretizations with high polynomial degree and regularity. In future work, we plan to study how to extend these adaptive primal spaces to isogeometric discretizations of the linear elasticity system, where we expect the adaptive selection of primal constraints for vector fields to be more challenging than in the scalar case. In particular, composite and almost incompressible materials might require new adaptive strategies in order to retain the BDDC scalability and robustness. An alternative approach for 
isogeometric discretizations of almost incompressible elasticity and Stokes problems, partly based on work by $\mathrm{Tu}$ and $\mathrm{Li}$ [41] has been recently explored in [36].

\section{REFERENCES}

[1] W.N. Anderson, JR., And R.J. Duffin, Series and parallel addition of matrices, J. Math. Anal. Appl., 26 (1969), pp. 576-594.

[2] S. Balay, S. Abhyankar, M.F. Adams, J. Brown, P. Brune, K. Buschelman, L. Dalcin, V. Eijkhout, W.D. Gropp, D. Kaushik, M.G. Knepley, L. Curfman Mcinnes, K. Rupp, B.F. Smith, S. Zampini, and H. Zhang, PETSc Web Page, 2015, http://www. mcs.anl.gov/petsc.

[3] L. Beirão da Veiga, A. Buffa, G. Sangalli, and R. Vazquez, Mathematical analysis of variational isogeometric methods, Acta Numer., 23 (2014), pp. 157-287.

[4] L. Beirão da Veiga, D. Cho, L.F. Pavarino, And S. SCACChI, Overlapping Schwarz methods for isogeometric analysis, SIAM J. Numer. Anal., 50 (2012), pp. 1394-1416, https://doi. org $/ 10.1137 / 110833476$.

[5] L. Beirão da Veiga, D. Cho, L.F. Pavarino, and S. Scacchi, BDDC preconditioners for isogeometric analysis, Math. Models Methods Appl. Sci., 23 (2013), pp. 1099-1142.

[6] L. Beirão da Veiga, D. Cho, L.F. Pavarino, and S. Scacchi, Isogeometric Schwarz preconditioners for linear elasticity systems, Comput. Methods Appl. Mech. Engrg., 253 (2013), pp. 439-454.

[7] L. Beirão da Veiga, L.F. Pavarino, S. Scacchi, O.B. Widlund, and S. Zampini, Isogeometric BDDC preconditioners with deluxe scaling, SIAM J. Sci. Comput., 36 (2014), pp. A1118-A1139, https://doi.org/10.1137/130917399.

[8] L. Beirão da Veiga, L.F. Pavarino, S. Scacchi, O.B. Widlund, and S. Zampini, BDDC deluxe for isogeometric analysis, in Proceedings of the Twenty Second International Conference on Domain Decomposition Methods, Lugano, Switzerland, 2013, Lecture Notes in Comput. Sci. Engrg. 104, Springer, New York, 2016, pp. 15-28.

[9] S.C. Brenner And L.-Y. Sung, BDDC and FETI-DP without matrices or vectors, Comput. Methods Appl. Mech. Engrg., 196 (2007), pp. 1429-1435.

[10] J.G. CAlvo AND O.B. Widlund, An adaptive choice of primal constraints for BDDC domain decomposition algorithms, Electr. Trans. Numer. Anal., 45 (2016), pp. 524-544.

[11] N. Collier, L. Dalcin, D. Pardo, and V.M. Calo, The cost of continuity: Performance of iterative solvers on isogeometric finite elements, SIAM J. Sci. Comput., 35 (2013), pp. A767-A784, https://doi.org/10.1137/120881038.

[12] J.A. Cottrell, T.J.R. Hughes, And Y. Bazilevs, Isogeometric Analysis. Towards Integration of $C A D$ and FEA, Wiley, New York, 2009.

[13] C. De Falco, A. Reali, and R. Vazquez, GeoPDEs: A research tool for isogeometric analysis of PDEs, Adv. Engrg. Softw., 42 (2011), pp. 1020-1034.

[14] C.R. Dohrmann, A preconditioner for substructuring based on constrained energy minimization, SIAM J. Sci. Comput., 25 (2003), pp. 246-258, https://doi.org/10.1137/ S1064827502412887.

[15] C.R. Dohrmann and C. Pechstein, Constraint and Weight Selection Algorithms for BDDC, talk by Dohrmann at Domain Decomp. Meth. Sci. Engrg. XXI, Rennes, France, 2012, http://www.osti.gov/scitech/servlets/purl/1117109.

[16] C.R. Dohrmann And O.B. WidLund, Some recent tools and a BDDC algorithm for 3D problems in H(curl), in Proceedings of the 20th International Conference on Domain Decomposition Methods, San Diego, CA, 2011, Lecture Notes in Comput. Sci. Engrg. 91, Springer, New York, 2013, pp. 15-26.

[17] C.R. Dohrmann And O.B. Widlund, A BDDC algorithm with deluxe scaling for threedimensional H(curl) problems, Comm. Pure Appl. Math., 69 (2016), pp. 745-770.

[18] C. Farhat, M. Lesoinne, P. LeTallec, K. Pierson, and D. Rixen, FETI-DP: A dual-primal unified FETI method-Part I. A faster alternative to the two-level FETI method, Internat. J. Numer. Methods Engrg., 50 (2001), pp. 1523-1544.

[19] K. Gahalaut, J. Kraus, and S. Tomar, Multigrid methods for isogeometric discretization, Comput. Methods Appl. Mech. Engrg., 253 (2013), pp. 413-425.

[20] K. Gahalaut, S.K. Tomar, and C.C. Douglas, Condition Number Estimates for Matrices Arising in NURBS Based Isogeometric Discretizations of Elliptic Partial Differential Equations, preprint, arXiv:1406.6808, 2014. 
[21] T.J.R. Hughes, J.A. Cottrell, And Y. Bazilevs, Isogeometric analysis: CAD, finite elements, NURBS, exact geometry, and mesh refinement, Comput. Methods Appl. Mech. Engrg., 194 (2005), pp. 4135-4195.

[22] H.H. KIM AND E.T. CHUng, A BDDC algorithm with enriched coarse spaces for twodimensional elliptic problems with oscillatory and high contrast coefficients, Multiscale Model. Simul., 13 (2015), pp. 571-593, https://doi.org/10.1137/140970598.

[23] H.H. KIm, E.T. ChUnG, AND J. WANG, BDDC and FETI-DP algorithms with adaptive coarse spaces for three-dimensional elliptic problems with oscillatory and high contrast coefficients, submitted; preprint at http://arxiv.org/abs/1606.07560, 2015.

[24] A. Klawonn, M. KüHn, and O. Rheinbach, Adaptive coarse spaces for FETI-DP in three dimensions, SIAM J. Sci. Comput., 38 (2016), pp. A2880-A2911, https://doi.org/10.1137/ $15 \mathrm{M} 1049610$.

[25] A. Klawonn, M. Lanser, P. Radtke, and O. Rheinbach, On an adaptive coarse space and on nonlinear domain decomposition, in Domain Decomp. Meth. Sci. Engrg. XXI, Rennes, France, 2012, Lecture Notes in Comput. Sci. Engrg. 98, Springer, New York, 2014, pp. $71-83$.

[26] A. Klawonn, P. Radtke, and O. Rheinbach, FETI-DP methods with an adaptive coarse space, SIAM J. Numer. Anal., 53 (2015), pp. 297-320, https://doi.org/10.1137/130939675.

[27] A. Klawonn, P. Radtke, And O. Rheinbach, FETI-DP with different scalings for adaptive coarse spaces, PAMM Proc. Appl. Math. Mech., 14 (2014), pp. 835-836.

[28] A. Klawonn, P. Radtke, and O. Rheinbach, A comparison of adaptive coarse spaces for iterative substructuring in two dimensions, Electron. Trans. Numer. Anal., 46 (2016), pp. $75-106$.

[29] A. Klawonn, O.B. Widlund, and M. Dryja, Dual-primal FETI methods for threedimensional elliptic problems with heterogeneous coefficients, SIAM J. Numer. Anal., 40 (2002), pp. 159-179, https://doi.org/10.1137/S0036142901388081.

[30] S.K. Kleiss, C. Pechstein, B. Jüttler, And S. Tomar, IETI-Isogeometric tearing and interconnecting, Comput. Methods Appl. Mech. Engrg., 247-248 (2012), pp. 201-215.

[31] J. Li And O.B. Widlund, FETI-DP, BDDC, and block Cholesky methods, Int. J. Numer. Methods Engrg., 66 (2006), pp. 250-271.

[32] J. Mandel AND C.R. Dohrmann, Convergence of a balancing domain decomposition by constraints and energy minimization, Numer. Linear Algebra Appl., 10 (2003), pp. 639-659.

[33] J. Mandel, C.R. Dohrmann, and R. Tezaur, An algebraic theory for primal and dual substructuring methods by constraints, Appl. Numer. Math., 54 (2005), pp. 167-193.

[34] J. Mandel, B. Sousedík, And J. Š́stek, Adaptive BDDC in three dimensions, Math. Comput. Simulation, 82 (2012), pp. 1812-1831.

[35] D.-S. Oh, O.B. Widlund, S. Zampini, and C.R. Dohrmann, BDDC Algorithms with Deluxe Scaling and Adaptive Selection of Primal Constraints for Raviart-Thomas Vector Fields, Technical Report TR2015-978, Courant Institute, NYU, New York, 2015.

[36] L.F. PaVArino AND S. SCACChi, Isogeometric block FETI-DP preconditioners for the Stokes and mixed linear elasticity systems, Comput. Methods Appl. Mech. Engrg., 310 (2016), pp. 694-710.

[37] C. Pechstein and C.R. Dohrmann, Modern Domain Decomposition Methods. BDDC, Deluxe Scaling, and an Algebraic Approach, seminar talk, Linz, 2013, http://people.ricam.oeaw. ac.at/c.pechstein/pechstein-bddc2013.pdf.

[38] C. Pechstein and C.R. Dohrmann, A Unified Framework for Adaptive BDDC, Technical Report 2016-20, Johann Radon Institute for Computational and Applied Mathematics (RICAM), University of Linz, 2016, http://www.ricam.oeaw.ac.at/files/reports/16/rep16-20. pdf.

[39] N. Spillane, V. Dolean, P. Hauret, P. Nataf, and J. Rixen, Solving generalized eigenvalue problems on the interface to build a robust two-level FETI method, C. R. Math. Acad. Sci. Paris, 351 (2013), pp. 197-201.

[40] A. Toselli And O.B. Widlund, Domain Decomposition Methods: Algorithms and Theory, Comput. Math. 34, Springer-Verlag, Berlin, 2004.

[41] X. TU AND J. LI, A FETI-DP type domain decomposition algorithm for three-dimensional incompressible Stokes equations, SIAM J. Numer. Anal., 53 (2015), pp. 720-742, https: //doi.org/10.1137/13094997X.

[42] O.B. Widlund And C.R. Dohrmann, BDDC deluxe domain decompositions, in Proceedings of the Twenty-second International Conference on Domain Decomposition Methods, Lugano, Switzerland, 2013, Lecture Notes in Comput. Sci. Engrg. 104, Springer, New York, pp. 93-103.

Copyright (c) by SIAM. Unauthorized reproduction of this article is prohibited. 
[43] S. ZAmPInI, PCBDDC: A class of robust dual-primal methods in PETSc, SIAM J. Sci. Comput., 38 (2016), pp. S282-S306, https://doi.org/10.1137/15M1025785.

[44] S. Zampini, Adaptive BDDC deluxe methods for H(curl), in Domain Decomposition Methods in Science and Engineering XXIII, Lecture Notes Comput. Sci. Engrg., Springer, New York, 2017, to appear.

[45] S. ZAMPINI AND D.E. KeyES, On the robustness and prospects of adaptive BDDC methods for finite element discretizations of elliptic PDEs with high-contrast coefficients, in Proceedings of the Platform for Advanced Scientific Computing Conference, ACM, New York, 2016, 6 .

[46] S. ZAMPINI AND X. TU, Adaptive multilevel BDDC deluxe algorithms for flow in porous media, submitted.

Copyright (c) by SIAM. Unauthorized reproduction of this article is prohibited. 